\title{
Corrigendum: Missing iris effect as a possible cause of muted hydrological change and high climate sensitivity in models
}

Thorsten Mauritsen and Bjorn Stevens

Nature Geoscience 8, 346-351 (2015); published online 20 April 2015; corrected after print 25 February 2016.

In the version of the Perspective originally published, there were errors in the Supplementary Information. After correcting these, the reported correlations between actual climate change feedback and tropical regression in the sentence beginning 'In the analysis of the CMIP5 ensemble presented here...' are +0.38 and +0.32 for the AMIP and historical experiments, respectively. In addition, the subsequent statement now reads: 'Of the eleven models that match CERES net regression in either experiment, four have ECS above $3 \mathrm{~K}$ and seven below. When run with a prescribed evolution of sea surface temperatures (AMIP) only the two versions of the Beijing Climate Center (BCC) model match observations in the slope of the regression between net, longwave and shortwave radiation with temperature. If run in coupled mode (historical) only one version of the Goddard Institute for Space Studies (GISS-E2-H) model matches CERES data.' The authors acknowledge David Coppin for pointing out these errors. These errors have been corrected in the online versions of the Perspective. 